\title{
A Multicriteria, Bat Algorithm Approach for Computing the Range Limited Routing Problem for Electric Trucks
}

\author{
JULIAN SCOTT YEOMANS (*) \\ OMIS Area, Schulich School of Business \\ York University \\ 4700 Keele Street, Toronto, ON, M3J 1P3 \\ CANADA
}

\begin{abstract}
As a result of increasing urban intensification, civic planners have devoted additional resources to more sustainability-focused logistics planning. Electric vehicles have proved to be both a lower cost alternative and more environmentally friendly than the more ubiquitous internal combustion engine vehicles. However, the predominant decision-making approaches employed by businesses and municipalities are not necessarily computationally conducive for the optimization and evaluation of urban transportation systems involving electric vehicles. An innovative modelling and planning approach is proposed to enable urban planners to more readily evaluate the contribution of electric vehicles in city logistics and to support the decision-making process. Specifically, this paper provides a multicriteria modelling-to-generate-alternatives (MGA) decision-support procedure that employs the Bat Algorithm (BA) metaheuristic for generating sets of alternatives for electric vehicle planning in urban transshipment problems. The efficacy of this multicriteria, BA-driven MGA approach for creating planning alternatives is demonstrated on an urban transshipment problem involving electric trucks.
\end{abstract}

Keywords: - Urban Logistics, Transshipment Problem, Bat Algorithm, Electric Vehicles, Multicriteria Objectives, Modelling-to-generate-alternatives

Received: November 4, 2020. Revised: May 11, 2021. Accepted: June 8, 2021. Published: June 15, 2021.

\section{Introduction to Urban Logistics and Electric Vehicles}

Resolving freight transshipment problems plays an important role in the sustainable development planning of large municipalities [1][2]. Effective urban transportation infrastructure must incorporate numerous incompatible components arising from structural deficiencies, vehicle congestion, high fuel usage, lack of human resources, and prevailing environmental restrictions [3]-[5]. A key sub-field in logistics planning has recently emerged to account for environmental constraints [6][7]. In addition to appeasing such sustainability requirements, urban freight carriers must also integrate low-cost, just-in-time transportation systems with high levels of end-user satisfaction [8].

Any logistics activities must simultaneously operate under the numerous limitations and constraints inherent within cities. As well as market-economic benefits, urban planning must enable social, sustainable, and environmental satisfaction [5][9]. A primary task uniting urban logistics with freight transport is to optimize logistical operations within the cities under social, environmental, energy usage, economic, traffic congestion, and financial constraints [2][3][5][8]. Without extensive logistics integration, civic planning cannot improve the overall quality of life. Consequently, effective urban logistics is a cornerstone of successful urban planning [6][10].

Street-level noise pollution when combined with vehicle emissions epitomize two principal environmental problem aspects inherent in urban logistics planning [3][5]. Although there has been a significant decrease in vehicle emissions due to more stringent government regulations, total emissions have actually increased due to even higher traffic volumes [1][11]. While rural air quality has improved, air quality has remained a major obstacle on urban roadways [12][13]. Consequently, supplementary actions need to be undertaken in order to improve the vehicle emission impacts on human health [5]. Noise pollution is also a major cost arising from transportation [14]. Heavy trucks, buses, diesel-engine cars, and motorcycles all contribute to elevated urban noise pollution. Replacing internal combustion engines (ICE) with silent-running, zero-emission electric motors affords one primary 
mechanism for overcoming excessive emissions issues and urban noise levels [12][13][15][16]. Recent developments in automotive technology and electric storage have resuscitated interest in using electric vehicles (EV) for urban freight delivery [15][17]. Relative to ICE vehicles, EVs possessing high efficiency electric motors hold numerous advantages such as low fuel and maintenance costs, zero emissions (improves air quality while decreasing emissions), and silent operations issues [9][15][16].

Disappointingly, when decisions between ICE and EV vehicles for urban transshipment are posed as strict either-or assessments, existing computational approaches establish ICE vehicles as the preferred choice. Within such decision-making contexts, the novel EV technologies currently lack widespread commercial viability due to limited vehicle ranges, lengthy recharging times, and significant initial implementation outlays [15][17]. Hence, the introduction of substantial technological developments is necessary prior to considering EVs as an effective replacement in long-range transportation [2]. Nevertheless, while it may not currently be feasible for EVs to act as a comprehensive logistical replacement for long-haul heavy-truck transportation, it can be demonstrated that the partial deployment of EVs in urban logistics can prove effective. Low operation costs can make EVs a promising alternative to ICE vehicles for within-city transportation purposes [1][12]. Specifically, while EVs may not appear feasible for ubiquitous transportation employment, when some routes can be electrified, it can be shown that significant cost reductions can be achieved. Therefore, municipalities and companies need to establish exactly which subsets of civic routes are most amenable to electrification. Under the appropriate circumstances, urban transshipment problems with electric vehicles can be transformed into determining which proportion of the transportation networks should be served by EVs and ICE vehicles, respectively, and identifying exactly which urban routings each vehicle-type covers. Determining solutions to this urban transshipment routing problem proves to be computationally complex.

Necessarily, "real world" municipal planning problems are frequently complex and contain numerous incompatible requirements that are not easily captured in the underlying mathematical models [18]. While "optimal" solutions can be calculated for the mathematical formulations, whether these answers produce best outcomes for the original "re- al" system is far less certain [19][20]. To improve decision-making under such ambiguities, it is often preferable to construct a limited number of dissimilar options that provide contrasting perspectives [21]. Preferably these alternatives should all possess good (i.e. near-optimal) objective measures with respect to the modelled objective(s), but be maximally different from each other in terms of the system structures characterized by their decision variables. Several approaches collectively referred to as modelling-to-generate-alternatives (MGA) have been developed in response to this multi-solution requirement [21]. The primary motive in MGA is to create a manageably small set of alternatives that are good with respect to all measured objective(s) yet are as fundamentally different as possible from each other within the prescribed decision space. By adopting a maximally different method, the resulting alternative solution set is likely to provide very different perspectives with respect to any unmodelled issues, while simultaneously providing different choices that all perform somewhat similarly with respect to the modelled objectives [21]. Decision-makers can then conduct a subsequent assessment of the alternatives to ascertain which specific option(s) most closely satisfies their underlying circumstances.

In this study, a mathematical model of the adapted urban transshipment problem with EVs is formulated [22] and a multicriteria MGA approach is developed to solve it. Because the actual optimization of this formulation can prove challenging, the computationally efficient Bat Algorithm (BA) metaheuristic is employed for its solution [23][24]. The computational effectiveness of this innovative MGA approach for solving the urban transshipment problem is established on an example of the range limited routing problem.

\section{Selection of Electric Vehicles}

Depending upon the specific configurations, the overall vehicle expenses of most EVs will tend to be $20 \%$ to $50 \%$ higher than ICE vehicles [18][22]. The increased costs arise mostly from the Lithium-ion batteries, although the costs of other components, especially the engines, are currently much higher than ICE motors. Conversely, as there is only a one rotating part in an electric motor in comparison to numerous moving parts in a combustion engine, the EV maintenance costs are approximately half those of ICE engines [15][17]. Moreover, EV fuel costs 
are considerably lower than ICE vehicles. Depending upon the fuel and electricity prices, cost per kilometre for EVs is from one half (USA) to one-sixth (Turkey). The environmental impact of EVs depends heavily on the generation source of electricity (e.g. wind, solar, hydroelectric, coal, nuclear). However, there is still an improvement in carbon emissions even when the electricity is sourced from coal. EVs area always far superior to ICE trucks with respect to air quality when measured at street level, [12].

The technology of ICE vehicles is far more versatile for long-range transportation purposes than that supplied by existing batteries. For example, petroleum-based vehicles can be refueled swiftly in less than 5 minutes and then be driven for distances of exceeding $500 \mathrm{~km}$. On the other hand, the batteries of a standard EV take more than an hour to recharge, providing only sufficient level, [ capacity to cover a range of no more than $100-150 \mathrm{~km}$. While swapping a battery or replenishing its electrolyte fluid provide two alternative approaches to the recharging speed dilemma, the battery distance capacity limitations do not change. Thus, for long range transportation needs, recharging points for EVs would need to be planned along the route to perhaps coincide with scheduled driver rest periods. The European Commission [4] restricts the longest nonstop period of truck driving to 4.5 hours which must then be followed by a requisite break of 45 minutes. Accordingly, to satisfy the EU driving requirements, long-range EV freight transportation would need to have a range of approximately $400 \mathrm{~km}$ together with a battery that could be fully charged within an hour. In such a situation, all travel and rest periods would have to be highly synchronized with battery recharging times, thereby introducing significant operational challenges and additional logistical planning in order to co-ordinate long-range transportation activities.

In major urban environments, EV distance limitations are a less challenging problem than the actual battery costs. For municipal logistics, EV recharging periods could occur during regular on-site loading/unloading activities or during pre-programmed periods of downtime (i.e. overnight). Regrettably for $\mathrm{EV}$, in large cities where $100^{+} \mathrm{km}$ routes would not be uncommon, the $100-150 \mathrm{~km}$ distance constraint would impose significant operational boundaries on the planning requirements. Additionally, to deliver refrigerated products, the overall distance limitations of EVs would be even more restricted due to the extra loading drawn from the batteries.

Consequently, if one were to pose the vehicle selection problem as a straightforward either-or option between EVs and ICE vehicles, current decision-making models would most likely result in the choice of ICE vehicles in urban transshipment. However, a different solution approach occurs when the problem becomes finding the optimal proportion of EVs to include in the fleet.

\section{Urban Transshipment with Electric Vehicles}

To facilitate a better understanding of the ensuing modelling, this section outlines the example of an easy-to-solve urban transshipment problem with electric vehicles described in [22]. Consider a symmetrical, urban distribution network containing a centralized storage warehouse, $A$, that supplies the four outlying facilities $B, C, D$, and $E$ (Figure 1). For simplicity, assume that the demand at each store is $1 \mathrm{unit} /$ day and that this quantity is delivered daily by a truck with a capacity of 2 units. Assume that the transportation and environmental costs are proportional to the distance covered and independent of the load amount. Hence, while in this problem, only operational costs are provided, in "real life", the calculations would also need to incorporate the full spectrum of expenses including environmental charges. From Figure 1, to satisfy the requisite transshipment requirements, it can be ascertained that the two feasible routes $A-E-A-C-A$ and $A-B-A-D-A$ would have a combined cost $160+160$ $=320$ units, while routes $A-D-C-A$ and $A-E-A-B-A$ would cost $180+120=300$ units. Nevertheless, it is relatively straightforward to determine that the minimum cost routes, $A-E-D-A$ and $A-C-B-A$, possess a total cost of $120+120=240$ units (see Table $1)$.

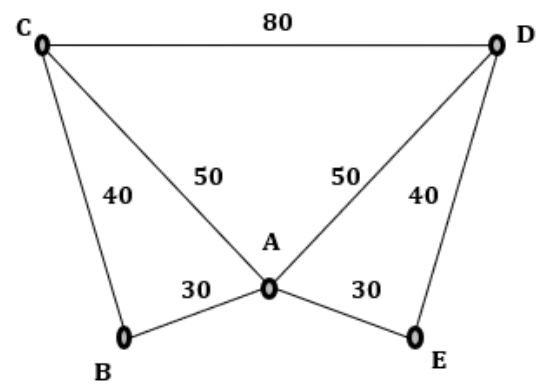

Figure 1. Sample Distribution Network (The numbers denote the distance or cost between nodes) 


\begin{tabular}{|c|c|l|}
\hline Solution & Distance & Truck Delivery Route \\
\hline $\mathbf{1}$ & 320 & $(A-B-A-D-A),(A-E-A-C-A)$ \\
\hline $\mathbf{2}$ & 300 & $(A-D-C-A),(A-B-A-E-A)$ \\
\hline $\mathbf{3}$ & 240 & $(A-E-D-A),(A-C-B-A)$ \\
\hline
\end{tabular}

Table 1. Possible Routes for the Network Transshipment Problem

If the entire truck fleet could be replaced by EVs with $1 / 6$ fuel costs, the minimum total cost would decrease to only $240 / 6=40$ units. However, if a reasonable cost electric truck typically possesses a $100 \mathrm{~km}$ range, then it would be neither operationally feasible to undertake $120 \mathrm{~km}(A-C-B-A, A-E-D-A)$ delivery routes, nor possible to even service the facilities $C$ and $D$. Due to these infeasibilities, if the problem were posed as whether the ICE trucks should be replaced by more energy efficient, environmentally benign EVs, the response would necessarily be negative.

However, if the problem was rephrased as finding "the ratio of the fleet to be converted to EVs" then this would lead to a completely different decision-problem. Specifically, the question could be re-stated as "in order to reduce overall costs, which routes could be satisfied by EVs and which routes would be served by ICE trucks?". In the transshipment network if nodes $B$ and $E$ were served by EVs, while $C$ and $D$ were still serviced by ICE trucks, then the optimal routes $A-E-A-B-A$ and $A-D-C-A$ would now have a total cost of 200 units $(180+$ 120/6) (see Table 2).

\begin{tabular}{|c|c|c|}
\hline Solution & Distance & Truck Delivery Route \\
\hline $\mathbf{1}$ & 200 & $(A-E-A-B-A),(A-D-C-A)$ \\
\hline
\end{tabular}

Table 2. Optimal Route for the Transshipment Problem with Partial EV Usage

Consequently, it can be clearly observed that a partial coverage of the distribution network using EVs would provide a better overall cost than the originally determined 240 units. In this situation, the urban transshipment problem with electric vehicles converts into a determination of what proportions of the network are served by EVs and ICE vehicles, and exactly which routes do these respective vehicle types need to cover. Optimizing this version of the urban transshipment problem with EVs will, henceforth, be referred to as the Range Limited Routing Problem (RLRP) [18][22].

\section{Range Limited Routing Problem Formulation}

The previously introduced, generalized mathematical formulation of the RLRP will be reviewed in this section [18][22]. In addition to challenges arising from the range limitations of EVs, the formulation of the RLRP combines standard vehicle routing problems [25] with capacitated vehicle routing problems [26].

The following notation will be employed in the RLRP [18]:

$k$-index for trucks $k=1, . ., K$.

$i, j-$ index for node (stores: $1, . ., N$ and warehouse: 0).

$R_{k}$ and $T_{k}$ - represents truck range and capacity, respectively, for truck $k, k=1, . ., K$.

$d_{0}-$ warehouse capacity.

$d_{i}$ - demand at node $i, i=1, . ., N$.

$r_{i j}$ - distance between node $i$ and $j, i, j=0, . ., N$.

$l_{i k}-$ load transshipped to node $i$ using truck $k$.

One assumption for the RLRP is that total capacity must equal the total demand with no excess capacity:

$$
\sum_{i=1+0 \mathrm{~K}} T_{k}=\sum_{i=1+\mathrm{N} N} d_{i}
$$

Hence, the complete mathematical programming formulation of the RLRP is to determine a solution to the following problem [22]:

$$
\operatorname{Max} \sum_{k=1 \text { ton }} \sum_{\text {i-oraV }} \sum_{j-i+1 \text { toN }} d_{j} x_{i j k}
$$

Subject to:

$$
\begin{aligned}
& \sum_{k=1+10 K} \sum_{j=1 \text { bN }} x_{0 j k} \leq K \\
& \sum_{i=0 \tan , i=j} x_{i j k}=\sum_{j-0 \operatorname{ton}: i=j} x_{i j k} \forall j, k \\
& \sum_{j=10 N, i=j} x_{i j k} \leq 1 \quad \forall i, k \\
& \sum_{\text {inlroNili=j }} x_{i j k} \leq 1 \quad \forall j, k \\
& \sum_{\text {imotal }} \sum_{j-\mathrm{OtoN}, i=j} r_{i j} x_{i j k} \leq R_{k} \quad \forall k \\
& l_{j k} \leq d_{j} \sum_{i=1+0 N_{i=j}} x_{i j k} \\
& \forall j, k \quad \text { (8) }
\end{aligned}
$$




$$
\begin{array}{ll}
\sum_{k-1+10 K} & l_{i k} \leq d_{i} \\
\sum_{i=1+10 N} & l_{i k} \leq T_{k}
\end{array}
$$$$
\forall i
$$

The constraints in the model ensure that at most $K$ trucks are used to deliver the goods (3), the truck routes are continuous and non-recurrent (4)-(6), the trucks do not exceed their range capacities (7), and that the demand at each store is satisfied by at least one truck (8)-(10). Determining a solution that satisfies all of the constraints enables a search for the feasibility of the urban transshipment problem with EVs. The optimal RLRP requires a feasible solution that maximizes the satisfied demand at each node and provides an urban cargo, fleet forming decision to the urban transshipment problem [18][22]. Optimizing the RLRP can prove very computationally challenging and the stability margins of related models are discussed more thoroughly in [25][26]. In the subsequent sections, the population-based, metaheuristic BA will be used to efficiently optimize the RLRP.

\section{Bat Algorithm Optimization}

This section provides a very brief overview of the BA procedure that is covered in significantly greater detail in [23][24]. The BA is a population-based metaheuristic. Each bat in the population corresponds to one potential solution to a problem and the initial population of fireflies is distributed randomly and uniformly through the decision space. The BA operates under the following three idealized: (i) All bats use echolocation to sense distance and can distinguish between food/prey and background barriers; (ii) Bats fly randomly with velocity $v_{i}$ at position $x_{i}$ with a fixed frequency $f_{\min }$ (or wavelength $\lambda$ ), varying wavelength $\lambda$ (or frequency $f$ ) and loudness $A_{0}$ to search for prey. They can automatically adjust the wavelength (or frequency) of their emitted pulses and adjust the rate of pulse emission $r$ within the range $[0,1]$ depending upon the proximity to their target; and, (iii) Although the loudness can vary in many ways, it can be assumed that loudness actually varies from a large (positive) $A_{0}$ down to a minimum value $A_{\min }$.

In the BA, the virtual bats are simulated. Certain rules are adopted to define how their positions $x_{i}$ and velocities $v_{i}$ in the d-dimensional search space are updated. The solutions/positions $x_{i y}$ and velocities $v_{i t}$ at time step $t$ are determined by: $f_{i}=f_{\text {min }}+\left(f_{\text {max }}-f_{\text {min }}\right) \beta$

$v_{i t}=v_{i t-1}+\left(x_{i y}-x_{*}\right) f_{i}$

$x_{i t}=x_{i t-1}+v_{i t}$

where $\beta$ is a random vector with each element generated from a uniform distribution within the range $[0,1]$. The value $x *$ is the current global best solution which is determined by comparing all the solutions among the $n$ bats in the $n$-dimensional solution vector $\boldsymbol{x}$. Initially each bat is assigned a random frequency drawn uniformly from the interval $\left[f_{\min }, f_{\max }\right]$. For the local search portion, once a solution is selected among the current best solutions, a new solution for each bat is generated locally using a random walk

$\boldsymbol{x}_{\text {new }}=\boldsymbol{x}_{\text {old }}+\varepsilon A_{t}$

where $\varepsilon$ is a random vector in the range [-1,1] and $A_{t}$ is the average loudness of all the bats at this time step, $t$.

The loudness $A_{i}$ and the rate of pulse emissions, $r_{i}$, have to be updated accordingly as the iterations proceed. As the loudness usually decreases once the bat has found its prey while the rate of pulse emissions increases, the loudness can be chosen as any value of convenience. For simplicity, one can use $A_{0}$ $=1$ and $A_{\text {min }}=0$, assuming that $A_{\text {min }}=0$ implies that a bat has just found the prey and temporarily stops emitting any sound. Thus,

$$
A_{i t+l}=\alpha A_{i t,} \quad r_{i t+1}=r_{i 0}[1-\exp (\gamma t)]
$$

where $\alpha$ and $\gamma$ are constants. The choice of parameters requires some experimenting, but in the simplest case $\alpha=\gamma$. Initially, each bat should have different values for their loudness and emissions rate, and this can be achieved via randomization. The loudness and emissions rates will only be updated by the algorithm if the new solutions are an improvement, which means that the bats are moving towards the optimal solution. The algorithm proceeds either until some convergence condition is achieved or for a maximum number of iterations [23][24]. Although neural networks [27] or other dynamical techniques could be considered for system optimization [28], a population-based solution algorithm is requisite in order to implement the subsequent MGA procedure. 


\section{Modelling to Generate Alternatives}

Mathematical programming has focused almost exclusively on finding single optimal solutions to single-objective problems or, equivalently, producing noninferior solutions to multi-objective formulations [20]. While these approaches may solve the formulations as constructed mathematically, whether these solutions are truly "best" for the original "real world" applications remains less certain [19]. In most "real world" systems, there are countless system specifications that can never be incorporated into the mathematical problem formulation [19]. Unavoidably, the majority of the subjective aspects remain unmodelled and unquantified in the mathematical system formulations. This frequently occurs when final outcomes are decided upon based not only on modelled objectives, but also on more subjective socio-political-economic preferences and stakeholder goals [23]. When unmodelled components are suspected to exist, non-traditional solution approaches are needed for searching the decision space not only for noninferior solutions, but also for sub-optimal possibilities. Specifically, any search for alternatives to problems suspected to possess unmodelled components must concentrate not only on a non-inferior set of solutions, but also necessarily on an explicit exploration of the problem's inferior solution space. Numerous "real life" instances of these types of modelling situations are illustrated in [19][20].

To demonstrate the impact of unmodelled objectives on a solution search, assume that the optimal solution to a maximization problem is $\boldsymbol{X}^{*}$ with objective value $Z 1^{*}$ [29]. Suppose a second, unmodelled, maximization objective $Z 2$ exists that represents some "politically acceptable" feature. Assume that the solution, $\boldsymbol{X}^{a}$, belonging to the 2-objective noninferior set, exists that corresponds to a best compromise solution if both objectives could actually have been simultaneously considered. While $\boldsymbol{X}^{a}$ would be the best solution to the real problem, in the actual mathematical formulation it would seem inferior to solution $X^{*}$, since $Z 1^{\text {a }} \leq Z 1^{*}$. Thus, when unmodelled components are included in the decision-making process, inferior decisions to the mathematically modelled system could actually be optimal to the fundamental "real" problem. If unmodelled aspects and unquantified objectives might exist, alternative solution procedures are essential to not only explore the decision region for noninferior solutions to the modelled problem, but also to concurrently search the decision space for explicitly inferior solutions.

Necessarily, then, in these situations, the aim is to create a workable set of options that are quantifiably good with respect to the modelled objectives yet are as different as possible from each other within the solution space. By satisfying this maximal difference condition, the resulting set of alternatives is able to supply truly different perspectives that all perform similarly with respect to the known modelled objective(s) yet very differently with respect to various potentially unmodelled aspects. By creating good-but-different options, the system designers are the able to consider potentially desirable qualities within the alternatives that might be able to satisfy the unmodelled objectives to varying degrees of stakeholder acceptability.

To motivate the process, it is necessary to formally characterize the mathematical definition of maximal difference [29]. Assume that the optimal solution to an original mathematical programming formulation is $\boldsymbol{X}^{*}$ with corresponding objective value $Z^{*}=F\left(X^{*}\right)$. An ensuing difference model can then be solved to produce an alternative solution, $\boldsymbol{X}$, that is maximally different from $\boldsymbol{X}^{*}$ :

$$
\begin{aligned}
& \text { Maximize } \Delta\left(\boldsymbol{X}, \boldsymbol{X}^{*}\right)={ }_{i}^{\operatorname{Min}_{i}}\left|X_{i}-X_{i}^{*}\right| \\
& \quad \text { Subject to: } \boldsymbol{X} \in D \\
& \left|F(\boldsymbol{X})-\boldsymbol{Z}^{*}\right| \leq T
\end{aligned}
$$

where $\Delta$ represents an appropriate difference function (shown in (16) as an absolute difference) and $T$ is a tolerance target relative to the original optimal objective value $Z^{*}$. $T$ is a user-specified limit that determines what proportion of the inferior region ought to be explored for acceptable alternatives. This difference function concept can be extended into a difference measure between any set of alternatives by replacing $X^{*}$ in the objective of the maximal difference model and calculating the overall minimum absolute difference (or some other function) of the pairwise comparisons between corresponding variables in each pair of alternatives subject to the condition that each alternative is feasible and falls within the specified tolerance constraint.

The population-based procedure that is subsequently employed is designed to generate a fixed, pre-determined number of close-to-optimal, but maximally different alternatives, by adjusting the value of $T$ and solving the corresponding maximal difference problem instance by exploiting the popu- 
lation structures of the optimization algorithm. The survival of solutions depends upon how well the solutions perform with respect to the problem's originally modelled objective(s) and simultaneously by how far away they are from all of the other alternatives generated in the decision space.

\section{Multicriteria MGA Procedure}

In this section, a data structure [21] is employed that permits a multicriteria MGA approach to be used for creating system options using any population-based solution algorithm [21] [29]-[33]. Suppose that it is desired to be able to produce $P$ alternatives that each possess $n$ decision variables and that the population algorithm is to possess $K$ solutions in total. Namely, each solution in the population contains one complete set of $P$ maximally different alternatives. Let $\boldsymbol{Y}_{k}, k=1, \ldots, K$, represent the $k^{\text {th }}$ solution consisting of one complete set of $P$ different alternatives. Specifically, if $\boldsymbol{X}_{k p}$ corresponds to the $p^{\text {th }}$ alternative, $p=1, \ldots, P$, of solution $k, k=$ $1, \ldots, K$, then $\boldsymbol{Y}_{k}$ can be represented as

$\boldsymbol{Y}_{k}=\left[\boldsymbol{X}_{k 1}, \boldsymbol{X}_{k 2}, \ldots, \boldsymbol{X}_{k P}\right]$.

If $X_{k j q}, q=1, \ldots, n$, is the $q^{\text {th }}$ variable in the $j^{\text {th }}$ alternative of solution $k$, then

$\boldsymbol{X}_{k j}=\left(X_{k j l}, X_{k j 2}, \ldots, X_{k j n}\right)$.

Accordingly, the entire population, $\boldsymbol{Y}$, comprised of $K$ different sets of $P$ alternatives can be expressed in vectorized format as,

$$
\boldsymbol{Y}^{\prime}=\left[\boldsymbol{Y}_{1}, \boldsymbol{Y}_{2}, \ldots, \boldsymbol{Y}_{K}\right] \text {. }
$$

The multicriteria method that follows can produce a pre-determined number of close-to-optimal, maximally different system options, by modifying the value of $T$ in the maximal difference model and using any population-based optimization algorithm to solve the corresponding, maximal difference problem. Each solution in the population is composed of one complete set of $P$ different possible system options [21]. By exploiting the co-evolutionary aspects of the algorithm, the procedure evolves each solution (i.e. set of alternatives) toward sets of dissimilar local optima within the solution domain. In this processing, each solution alternative mutually experiences the search steps of the algorithm. Solution survival depends both upon how well the solutions perform with respect to the modelled objective(s) and by how far apart they are from every other alternative in the decision space.

A straightforward process for generating alternatives solves the maximum difference model iteratively by incrementally updating the target $T$ when- ever a new alternative needs to be produced and then re-solving the resulting model [29]. However, in the following MGA algorithm, each solution in the population contains exactly one entire set of alternatives and the maximal difference is calculated only for that particular solution (i.e. the specific alternative set contained within that solution in the population). Hence, by the evolutionary nature of the population-based search procedure, in the subsequent approach, the maximal difference is calculated simultaneously for the specific set of alternatives considered within each specific solution - and the need for concurrent subpopulation aggregation measures is circumvented.

Using the data structure terminology, the steps for the multicriteria MGA algorithm are as follows [21] [24]-[26][29]-[33]. The stratification approach employed by this method can be easily modified for solution via any population-based optimization algorithm.

Preliminary Step. Solve the original optimization problem to find its optimal solution, $X^{*}$. Based upon the objective value $F\left(X^{*}\right)$, establish $P$ target values. $P$ represents the desired number of maximally different alternatives to be generated within prescribed target deviations from the $X^{*}$. Note: The value for $P$ must be fixed a priori by the decision-maker.

Without loss of generality, it is possible to forego this step and to use the algorithm to find $X^{*}$ as part of its solution processing in the subsequent steps. However, this significantly increases the number of iterations of the computational procedure and the initial stages of the processing become devoted to finding $X^{*}$ while the other elements of each population solution are retained as essentially "computational overhead".

Step 1. Create an initial population of size $K$ where each solution is divided into $P$ equally-sized partitions. The partition size corresponds to the number of decision variables in the original optimization problem. $\boldsymbol{X}_{k p}$ represents the $p^{\text {th }}$ alternative, $p$ $=1, \ldots, P$, in solution $\boldsymbol{Y}_{\boldsymbol{k}}, k=1, \ldots, K$.

Step 2. In each of the $K$ solutions, evaluate each $\boldsymbol{X}_{k p}, p=1, \ldots, P$, with respect to the modelled objective. Alternatives meeting both their target constraint and all the other problem constraints are designated as feasible, while all other alternatives are designated as infeasible. An individual solution can only be designated as feasible if all of the alternatives contained within it are feasible.

Step 3. Apply an appropriate elitism operator to each solution to rank order the best individuals in 
the population. The best solution is the feasible solution containing the most distant set of alternatives in the decision space (the distance measures are defined in Step 5).

Note: Because the best-solution-to-date is always retained in the population throughout each iteration, at least one solution will always remain feasible. Furthermore, a feasible solution based on the initialization step can be constructed using $P$ repetitions of $\boldsymbol{X}^{*}$.

Step 4. Stop the algorithm if the termination criteria (such as maximum number of iterations or some measure of solution convergence) are met. Otherwise, proceed to Step 5.

Step 5. For each solution $\boldsymbol{Y}_{k}, k=1, \ldots, K$, calculate $R$ Max-Min and/or Max-Sum distance measures, $D_{k, r}^{r}=1, \ldots, R$, between all of the alternatives contained within the solution.

As an illustrative example for calculating the multicriteria distance measures, compute:

$$
\begin{aligned}
& D_{k}^{l}=\Delta^{1}\left(\boldsymbol{X}_{k a}, \boldsymbol{X}_{k b}\right)={ }_{a, b q}^{\text {Min }}\left|X_{k a q}-X_{k b q}\right|, \\
& \quad a=1, \ldots, P, b=1, \ldots, P, q=1, \ldots, n, \\
& D^{2}{ }_{k}=\Delta^{2}\left(\boldsymbol{X}_{k a}, \boldsymbol{X}_{k b}\right) \\
& =\sum_{a=1 \neq P} \sum_{b-1+p)} \sum_{q-1 \ldots n}\left|X_{k a q}-X_{k b q}\right| .
\end{aligned}
$$

and

$$
\begin{aligned}
& D^{3}{ }_{k}=\Delta^{3}\left(\boldsymbol{X}_{k a}, \boldsymbol{X}_{k b}\right) \\
& \quad \sum_{a=1 \neq p} \sum_{b-1 ; \circ p} \sum_{q-1 \ldots n}\left(X_{k a q}-X_{k b q}\right)^{2} .
\end{aligned}
$$

$D^{I}{ }_{k}$ denotes the minimum absolute distance, $D^{2}{ }_{k}$ represents the overall absolute deviation, and $D^{3}{ }_{k}$ determines the overall quadratic deviation between all of the alternatives contained within solution $k$.

Alternatively, distance function could be calculated using other appropriately defined measures.

Step 6. Let $D_{k}=G\left(D^{l}{ }_{k}, D^{2}{ }_{k}, D^{3}{ }_{k}, \ldots, D^{R}{ }_{k}\right)$ represent the multicriteria objective for solution $k$. Rank the solutions according to the distance measure $D_{k}$ objective - appropriately adjusted to incorporate any constraint violation penalties for infeasible solutions. The goal of maximal difference is to force alternatives to be as far apart as possible in the decision space from the alternatives of each of the partitions within each solution This step orders the specific solutions by those solutions which contain the set of alternatives which are most distant from each other.
Step 7. Apply appropriate metaheuristic "change operations" to each solution within the population and return to Step 2.

\section{Computing Solutions to the Range Limited Routing Problem with the MGA Algorithm}

In this section, a computational testing of the efficacy of solving the RLRP using the BA-driven MGA procedure will be illustrated. As described above, decision-makers frequently prefer to be able to select from a contrasting set of "near-optimal" alternatives that significantly differ from each other in terms of the system structures characterized by their decision variables. In order to create a set of maximally different alternative planning options, a computational testing of the efficacy of employing the MGA algorithm will be illustrated on the RLRP introduced earlier.

Suppose that, together with determining the optimal solution, there is also a desire to construct an additional 3 maximally different solution alternatives that possess objective values within $15 \%$ of optimal. Since the example problem is small, assume that the direction of travel specified in the distribution network is considered non-commutative (i.e. the route $A-B-C-A$ is considered to be different from the route $A-C-B-A)$. Under these problem specifications, the RLRP of the example problem was solved by the BA-driven MGA algorithm create the 4 maximally different solutions shown in Table 3 . It can be explicitly noted that the best solution calculated by the BA procedure is identical to the optimal solution previously found in the RLRP example section.

\begin{tabular}{|c|c|c|}
\hline Alternatives & $\mathbf{F}(\boldsymbol{X})$ & $\boldsymbol{X}$ (Truck Routes) \\
\hline Best Found & 200 & $(A-E-A-B-A),(A-D-C-A)$ \\
\hline $\mathbf{1}$ & 200 & $(A-B-A-E-A),(A-C-D-A)$ \\
\hline $\mathbf{2}$ & 240 & $(A-B-C-A),(A-E-D-A)$ \\
\hline $\mathbf{3}$ & 240 & $(A-C-B-A),(A-D-E-A)$ \\
\hline
\end{tabular}

Table 3. Objective Values and Solutions for the 4 Maximally Different Alternatives in the RLRP

The computational example illustrates how the MGA modelling perspective can be effectively used to generate multiple, good solution alternatives by employing the very computationally efficient, FA-based metaheuristic. By following this process, the alternatives produced all satisfy the required 
system criteria, yet remain as maximally different from each other as possible within the decision space. Furthermore, the MGA procedure has simultaneously performed exceedingly well with respect to its role in function optimization. It can be noted explicitly that the overall best solution calculated by the MGA procedure is identical to the optimal solutions determined earlier for the RLRP example.

As described earlier, the selection of EVs for support in urban transshipment planning cannot necessarily be stated in either-or terms with respect to the replacement of ICE trucks with EVs. Consequently, in addition to calculating the optimal proportion of EVs relative to ICE vehicles needed to service an urban logistics network, the RLRP model was formulated to also determine the optimal, specific truck routes for each type of vehicle. Given the levels of uncertainty in the RLRP, it is actually preferable to create several quantifiably good alternatives that concurrently provide very different perspectives to the potentially unmodelled performance. The unique performance features captured within these dissimilar alternatives can result in very different system performance with respect to the unmodelled issues, thereby incorporating the unmodelled issues into the actual solution process. The computational results from the RLRP urban transshipment problem demonstrate that the BA-driven MGA algorithm provides a suitable approach for producing not only a requisite set of maximally different alternatives but also the overall single optimal solution of the problem formulation should that result, alone, be desired.

In overall summary, this computational testing reinforces a number of important characteristics of an effective MGA procedure. Specifically, the evolutionary features of the BA actually generate more good planning options than decision-makers would be able to create using other MGA techniques because of the dynamic nature of the BA's population-based search structure. By the inherent design of the MGA algorithm, the alternatives produced are good for planning purposes since all of their structures are maximally different from each other. Finally, the method is extremely efficient computationally as it requires only a single run to compute its complete set of alternatives. Specifically, in order to construct $n$ different solution alternatives, the MGA algorithm needs to run exactly once irrespective of the magnitude of $n$.

\section{Conclusion}

Complex planning inherently involves complicated performance components that can be confounded by incongruent requirements and inconsistent performance objectives. These decision environments frequently contain incompatible design specifications that are problematic - if not impossible - to incorporate when ancillary decision support models are constructed. Invariably, there are unmodelled elements, not apparent during model formulation, that can significantly affect solution adequacy. These confounding features require the decision-makers to integrate numerous discrepancies into their solution processes before a definitive solution can be determined. Faced with such inconsistencies, it is unlikely that any single solution can simultaneously satisfy all ambiguous system requirements without significant compromises. Under such circumstances, it is desirable for decision support approaches to somehow address these complicating features, while simultaneously being flexible enough to capture the potential planning incongruities. Planning for logistical aspects of the urban transshipment using electric trucks provides one such example of this decision-making environment.

Although electric vehicles cannot provide a direct substitute to the logistical requirements of long-haul heavy-truck transportation, ample room does exist to accommodate them in urban logistics. The decision to employ electric vehicles can be a strict "either-or" assessment or, alternatively, the ratio of electrification required in covering a partial subset of the urban routes. While a total adoption of EVs may not be feasible, in general, when some portion of the routes can be electrified, there may be significant potential for overall cost reductions. Therefore, to decrease costs, municipalities and companies would need to identify which routes are most conducive to electrification.

While the RLRP formulation articulates the problem, determining its solution remains challenging. Namely, a computationally efficient solution approach is required. This paper has employed the computationally efficient BA-based MGA procedure to enable this solution determination. The computational efficacy of employing the algorithm in conjunction with the population-based BA metaheuristic was demonstrated on the urban transhipment RLRP example. The computational procedure not only produced a set of high-quality, maximally different solution alternatives, but also simultaneously found the optimal solution to the 
formulated RLRP problem. The practicality of this BA-based MGA solution approach for urban transhipment problems using EVs can clearly be extended to wide range of other "real world" applications. Furthermore, computational comparisons from implementing the MGA procedure using alternate population-based meta-heuristics would provide one avenue for further study. Additional evaluations as to the nature of the specific multi-objective measures employed in the MGA procedure when applied under different circumstances are warranted. Such extensions will be considered in forthcoming research.

\section{References:}

[1] C. Thiel, W. Nijs, S. Simoes, J. Schmidt, A. van Zyl, and E. Schmid, The Impact of the EU Car CO2 Regulation on the Energy System and the Role of Electro-Mobility to Achieve Transport Decarbonisation, Energy Policy, Vol. 96, 2016, pp. 153-166.

[2] S. Winkelhaus, and E.H. Grosse, Logistics 4.0: A Systematic Review Towards a New Logistics System, International Journal of Production Research, Vol. 58, No. 1, 2020, pp. 18-43.

[3] A. De Marco, G. Mangano, and G. Zenezini, Classification and Benchmark of City Logistics Measures: An Empirical Analysis, International Journal of Logistics Research and Applications, Vol. 21, No. 1, 2018, pp. 1-19.

[4] European Commission. Panorama of Transport, $2009 . \quad$ Retrieved from https://ec.europa.eu/eurostat/documents/321749 4/5711595/KS-DA-09-001-EN.PDF/9c90d4895009-4acc-9810-ae39612897d3.

[5] M. Kiba-Janiak, and J. Witkowski, Sustainable Urban Mobility Plans: How Do They Work?, Sustainability, Vol. 11, No. 17, 2019, pp. 4605.

[6] E. Taniguchi, Concepts of City Logistics for Sustainable and Liveable Cities, Procedia - Social and Behavioral Sciences, Vol. 151, No. 10, 2014, pp. 310-317.

[7] UNESCAP. Commercial Development of Regional Ports as Logistics Centres. United Nations. Economic and Social Commission for Asia and the Pacific (UNESCAP), 2003. Retrieved from https://www.unescap.org/sites/default/files/pub 2194 fulltext.pdf.

[8] $\bar{E}$. Taniguchi, R.G. Thompson, T. Yamada, and R. van Duin, City Logistics, Holland: Pergamon, 2001.
[9] D.B. Richardson, Electric Vehicles and the Electric Grid: A Review of Modeling Approaches, Impacts, and Renewable Energy Integration, Renewable and Sustainable Energy Reviews, Vol. 19, No. 3, 2013, pp. 247-254.

[10] E. Taniguchi, and R.E.C.M. van der Heijden, An Evaluation Methodology for City Logistics, Transport Reviews, Vol. 20, No. 1, 2000, pp. 65-90.

[11] EEA Report, No. 3/2004, Ten Key Transport and Environment Issues for Policy-Makers, Copenhagen: European Environment Agency, 2004.

[12] M. Hernandez, M. Messagie, O. Hegazy, L. Marengo, O. Winter, and J. Van Mierlo, Environmental Impact of Traction Electric Motors for Electric Vehicles Applications, The International Journal of Life Cycle Assessment, Vol. 22, No. 1, 2017, pp. 54-65.

[13] R.N. Tuncay, and Ö. Üstün, Otomativ Sektör Kurulu Raporu: Elektrikli Araçlarda Geçmişten Geleceğe Bakış, MÜSİAD, 2012.

[14] European Commission. Road Transport: Reducing CO2 Emissions from Vehicles, 2016. Retrieved from http://ec.europa.eu/clima/policies/transport/vehi cles/index_en.htm.

[15] S. Khemakhem, M. Rekik, and L. A. Krichen, Flexible Control Strategy of Plug-In Electric Vehicles Operating in Seven Modes for Smoothing Load Power Curves in Smart Grid, Energy, Vol. 118, 2017, pp. 197-208.

[16] M. Weiss, M.K. Patel, M. Junginger, A. Perujo, P. Bonnel, and G. Grootveld, On the Electrification of Road Transport - Learning Rates and Hybrid-Electric and Battery Electric Vehicles, Energy Policy, Vol. 48, No., 20129, pp. 374-393.

[17] A.S. Brower, T. Kuramochi, M. Broek, and A. Faaij, Fulfilling the Electricity Demand of Electric Vehicles in the Long Term Future: An Evaluation of Centralized and Decentralized Power Supply Systems, Applied Energy, Vol. 107, No. 1, 2013, pp. 33-51.

[18] G. Gunalay, and J. Yeomans, An Innovative Modelling and Decision-Support Approach for Evaluating Urban Transshipment Problems Using Electrical Trucks, International Journal of Smart Vehicles and Smart Transportation, Vol. 3, No. 2, 2020, pp. 19-37.

[19] M. Brugnach, A. Tagg, F. Keil, and W.J. De Lange, Uncertainty matters: computer models at the science-policy interface, Water $R e$ sources Management, Vol. 21, 2007, pp. 1075-1090. 
[20] J.A.E.B. Janssen, M.S. Krol, R.M.J. Schielen, and A.Y. Hoekstra, The effect of modelling quantified expert knowledge and uncertainty information on model-based decision making, Environmental Science and Policy, Vol. 13, No. 3, 2010, pp. 229-238.

[21] G. Gunalay, and J. Yeomans, Multicriteria Generation of Alternatives for Engineering Optimization Problems Using Population-Based Metaheuristics: A Computational Test, WSEAS Transactions on Computers, Vol. 18, No. 31, 2019, pp. 239-247.

[22] Y. Gunalay, and J.S. Yeomans, An Algorithm for Computing Solutions to the Range Limited Routing Problem Using Electrical Trucks, WSEAS Transactions on Computers, Vol. 19, No. 7, 2020, pp. 47-53.

[23] X.S. Yang, A New Metaheuristic Bat-Inspired Algorithm, in J.R. Gonzalez (Ed.), Nature Inspired Cooperative Strategies for Optimization (NICSO 2010), Springer, New York, 2010, pp. 65-74.

[24] X.S. Yang, Nature-Inspired Metaheuristic Algorithms 2nd Ed., Frome (UK): Luniver Press, 2010.

[25] S.N. Kumar, and R. A Panneerselvam, Survey on the Vehicle Routing Problem and Its Variants. Intelligent Information Management, Vol. 4, No. 3, 2012, pp. 66-74.

[26] P. Toth, and D. Vigo, The Vehicle Routing Problem, USA: Society for Industrial and Applied Mathematics, 2002.

[27] A. Maniatopoulos, A. Gazis, V.P. Pallikaras, and N. Mitianoudis, Artificial Neural Network Performance Boost using Probabilistic Recovery with Fast Cascade Training, International Journal of Circuits, Systems and Signal Processing, Vol. 14, 2020, pp. 847-854

[28] V. Poliakov, The Artificial Intelligence and Design of Multibody Systems with Predicted Dynamic Behavior, International Journal of Circuits, Systems and Signal Processing, Vol. 14, 2020, pp. 972-977.

[29] J.S. Yeomans, An Algorithm for Generating Sets of Maximally Different Alternatives Using Population-Based Metaheuristic Procedures, Transactions on Machine Learning and Artificial Intelligence, Vol. 6, No. 5, 2018, pp. 1-9.

[30] J.S. Yeomans, A Bicriterion Approach for Generating Alternatives Using Population-Based Algorithms, WSEAS Transactions on Systems, Vol. 18, No. 4, 2019, pp. 29-34.

[31] J.S. Yeomans, A Simulation-Optimization Algorithm for Generating Sets of Alternatives Using Population-Based Metaheuristic Proce- dures, Journal of Software Engineering and Simulation, Vol. 5, No. 2, 2019, pp. 1-6.

[32] J.S. Yeomans, A Stochastic, Dual-Criterion, Simulation-Optimization Algorithm for Generating Alternatives, Journal of Computer Science Engineering, Vol. 5, No. 6, 2019, pp. $1-10$.

[33] J.S. Yeomans, A Multicriteria Simulation-Optimization Algorithm for Generating Sets of Alternatives Using Population-Based Metaheuristics, WSEAS Transactions on Computers, Vol. 18, No. 9, 2019, pp. 74-81.

Contribution of Individual Authors to the Creation of a Scientific Article (Ghostwriting Policy)

The author contributed in the present research, at all stages from the formulation of the problem to the final findings and solution.

Sources of Funding for Research Presented in a Scientific Article or Scientific Article Itself

No funding was received for conducting this study.

\section{Conflict of Interest}

The author has no conflict of interest to declare that is relevant to the content of this article.

Creative Commons Attribution License 4.0 (Attribution 4.0 International, CC BY 4.0)

This article is published under the terms of the Creative Commons Attribution License 4.0 https://creativecommons.org/licenses/by/4.0/deed.en US 